\title{
A Component-Based Reflective Middleware Approach to Context-Aware Adaptive Systems
}

\author{
Zhang Kuo, Wu Yanni, Zheng Zhenkun, Wang Xiaoge, and Chen Yu \\ Department of Computer Science and Technology \\ Tsinghua University, Beijing 100084, China \\ \{zhangkuo99, wuyanni98, zhengzhenkun98\}@mails.tsinghua.edu.cn \\ \{wangxg,yuchen\}@mail.tsinghua.edu.cn \\ http://os.riit.tsinghua.edu.cn
}

\begin{abstract}
In ubiquitous computing environment, a middleware abstracting a unified service for various types of applications operating over a static environment is not valid; in particular, ubiquitous applications does not benefit from dynamic reconfiguration and adaptive computing. In this paper, we introduce our reflective middleware platform PURPLE ${ }^{1}$, which is designed and implemented to fit the varying requirement of ubiquitous computing: such as adaptability, configurability, context awareness.
\end{abstract}

\section{Introduction}

Ubiquitous(or Pervasive) computing focuses on integrating computers with the physical environments, to make computing and communication essentially transparent. Devices that operate in these environments are usually embedded and use lowpower wireless communication means; topologies of the networks are dynamic due to arbitrary node mobility, and usually contain no dedicated network connectivity devices. So ubiquitous applications should address the characteristics: such as context awareness and dynamic configurations.

We argue that these two characteristics specify the need for system support, while not application-specific, for both development and runtime services in ubiquitous environments. In this respect, middleware-oriented approaches can be very effective if they can reduce the effort required to develop ubiquitous software and provide appropriate runtime services for applications with the two characteristics above. However, most current middleware solutions are not adequate to support contextawareness and dynamic reconfigurations for real-time. Moreover, most currently available middleware for ubiquitous computing, are based on existed componentplatforms, which are modular but static; it means that middleware developers need to pay additional attention to code reuse and leveraging existing systems, as well as adding features to make the system more flexible.

Research on reflective middleware has a history of ten years. Earlier projects mostly concentrated on what a real reflective middleware should be like and how to

1 Supported by the National High-Tech Research and Development Plan of China (No.2003AA1Z2090), National Natural Science Foundation of China (No. 60203024), and

Tsinghua University Research Found (No. JC2003021) 
build it, such as Open ORB [1], OpenCorba, mChaRM; Recent projects usually have more explicit application background including reflective middleware for pervasive/ubiquitous computing and mobile computing. Some significant projects are LegORB [3], Chisel, CARISMA, ReMMoC [5]. The applications of reflective middleware cover almost every hot research area in recent years, such as component based OS [4] P2P, and Grid computing and programmable networking [2], etc. The results of the research in reflection are also being incorporated in industrial-strength middleware, such as JBoss 4.0.

In this paper, we present a reflective middleware, which is based on EFL component platform, to facilitate the applications development in ubiquitous computing. It has the features of context-aware, dynamic-configurable and policy-driven to fit the requirement of constantly changed context. Unlike most adaptive middleware, it supports dynamic reconfiguration at both middleware and application layers by the help of reflection. We also innovatively present a multilevel Meta data, which reflects information and capabilities of the system from different perspectives with different resolution. An abstraction of components' interactions and dynamic reconfigurations is expressed in a high-level declarative language written in XML which is customizable and extendable.

\section{Reflections in EFL Component Platform}

EFL is a lightweight, efficient and reflective component platform running on Linux developed by our group. It is written in $\mathrm{C}$ for best performance and minimum memory footprint. Its specification adopts the design of Microsoft's DCOM, such as binary level interoperability standard, transparent local/remote invocations. And the platform deploys a standard runtime substrate that manages the running context of an EFL component. Many new features are brought into this component model. Our middleware is built upon on EFL component platform, so we focus on the implementation of reflection on the substrate of the system. During the study of the design and implementation of reflective middleware, various possibilities of constructing metalevel are investigated. Finally, an innovative multi-levels reflection model is proposed.

This model provides fine-grained metadata from different levels of a program. It can reflect information and capabilities of the system from different sources with different resolving power. In this novel multi-level reflection model, the metadata is distributed on three levels: the Interface-level, the Component-level and the Middleware-level. They are used to reflect the base level information in different perspectives and granularities. The first two levels are directly supported by EFL. The fundamental concepts of an EFL component are interfaces; Interface-level Meta data contains the definition of interfaces, functions and parameters. It provides the finest grain of self-description information of the system, and it is obtained by using the IMetaInterface interface of EFL component. With the help of this, middleware can dynamically load components and invoke the method without generating accessing code. Component-level Meta data is used to describe an EFL component's requirement of context for running (such as hardware and OS requirement). The Meta data in this level also contains the info of the reliance of dependency that one component lies on others. Component-level Meta data can be accessed by ICompMetaInterface 
interface of EFL component. The Middleware-level Meta data describes a graph of runtime components on middleware platform, and provide the dynamic reconfiguration strategy.

\section{Middleware System Overview}

\subsection{Architecture}

The architecture of PURPLE is shown in Figure 1. Firstly, we will present an overview of PURPLE in this section.

Applications

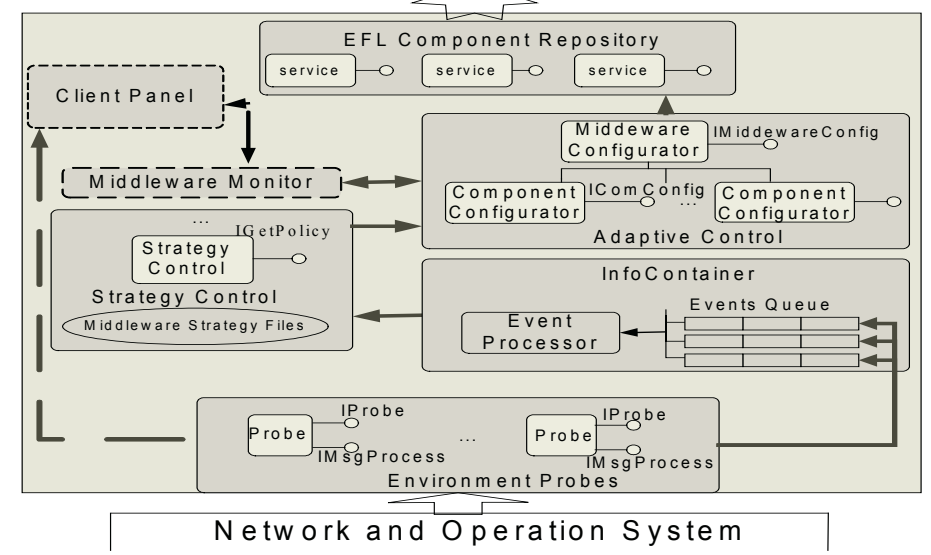

Fig. 1. The Architecture of Reflective Middleware

The PURPLE programming model is based upon the concept of described abstract services, such as WSDL. The underlying concrete implementations are supplied by groups of service components in EFL Component Repository Module. The tools for maintaining the repository is provided, so that developers can enrich the repository to meet applications' requirement. The components in this repository are classified as base-level components.

The support to the dynamic adaptation of the base-level components is offered by Adaptive Control Module. This module functions as the controller of Components' Runtime view; in other words, it is the graph of runtime components, the most important part of Middleware-level Meta data. Dynamic reconfiguration strategy is another part of Middleware-level Meta data. This information is maintained by Strategy Control Module. The strategies are recorded in strategy files and describe the actions should be taken when context value matches. The context specifications are presented in section 3.3, developers can customized their own strategy files according to the Schema we provide. InfoContainer Module is in charge of environment information analyzing and events maintenance and dispatching. Environment information includes various changes in network status, machine resources and connectivity status. Details are demonstrated in section 3.2. Environment Probes Module makes a real time collection of platform environment information, such as CPU usage, Battery 
info, Network info, etc. It provides the context-aware ability and raises a series of events to Event processor. Probes can be reconfigured and deployed into Probes container dynamically. So it is easily extendable in future.

Client Panel and Middleware Monitor are drawn in broken line in figure 1, that's because these two modules are optional in our platform. Client Panel can provide the visualization and runtime configuration of the middleware structure. It collects system info through Middleware Monitor, and represents the running context info by subscribing message from Environment Probes. Middleware Monitor interacts with Adaptive Control Module, and becomes a channel between Client Panel and other Middleware Modules.

\subsection{Context-Awareness}

The detailed design of InfoContainer is shown in Figure 2. PURPLE achieves context-awareness and policy-driven by using an Event Trigger Model. Event Trigger Model supports subscription from all entities within the PURPLE platform, including system components (such as component for strategy control), base-level components and applications as well.

The Event Module in Figure 2 represents the entity which is interested in special contexts. Each module is a composite of Receivers, which are EFL components implementing IClientSink interface. Selected methods in IClientSink interfaces are registered to a customizable Event Container, and will be invoked when interested contexts meet. Event Container is also an EFL component; it maintains the connections from all Event Module and triggers the subscribed events at right time. Each Event Container subscribes several interested context info to Event Processor. When context information comes to InfoContainer, Events Queue module unmarshals the message and filters the useless information, and then useful context information is classified, cashed and dispatched to Event Processor immediately. Event Processor takes care of the activity of each Event Container and injects the subscribed context info into specified Event Containers.

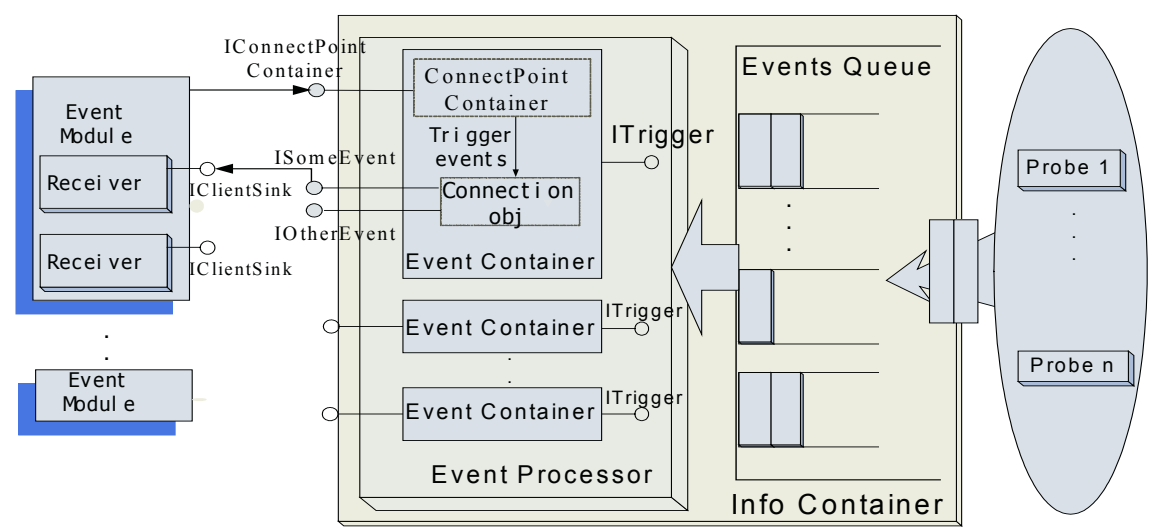

Fig. 2. The structure of InfoContainer 
There are two problems exist. Firstly, large amount of events can be triggered simultaneously when context changes obviously. The property of priority for each Event Module is added; this priority is registered to its Event Container and maintained by Event Processor, so that concurrent events can be triggered orderly according to their priority. Secondly, the Events Containers can be customized by developers to subscribe variety of context information, but it can't be deployed dynamically without compiling; further more, composition of primitive context to form a hierarchical context is needed in system adaptation. We achieve these by introducing Strategy Control Module. Strategy files are maintained by this module; these files are written in XML format, providing the direction of system adaptation when context changed (detail of this is discussed in section 3.3), and these files can be deployed into Strategy Control Module on the fly, too.

The context specifications in strategy files are presented in next section.

\subsection{Context Specification for Policy-Driven}

Using the strategy file, the context-awareness of the adaptive system can be expressed by associating context expressions along with the application-level behavior. The elements of strategy file are presented in the following part. Context Items are expressions that return Boolean values which indicate different situations in specific context. For example, a context item $\mathrm{C} 1$ can refer to a $0 \%$ 50\% CPU usage expression as $\mathrm{C} 1=\left(0<=\mathrm{CPU} \_\right.$Usage $\left.<=0.5\right)$, the value of $\mathrm{C} 1$ is either true or false; and $\mathrm{C} 2$ may represent the equipment used in a Cellular Network Context ( $\mathrm{C} 2=$ (NetworkContext $==$ GPRS ). Context Operators are operators to specify the relationships among multiple Context Items. Context Expressions are expressions to represent the relations among context items using context operators. (Such as $\mathrm{C} 1 \& \mathrm{C} 2$ specifies a specific context).

The operators can be used to specify the relations between Context Items or Expressions. In this sense, we can define a complex expression recursively by using simpler expression. For example: $(\mathrm{C} 1->\mathrm{C} 2)$ in $\mathrm{t}$ represents that $\mathrm{C} 2$ becomes true within the time $\mathrm{t}$ until $\mathrm{C} 1$ being true; $((\mathrm{C} 1 \mid \mathrm{C} 2) \&(\sim \mathrm{C} 3))$ in $\mathrm{t}$ means that $\mathrm{C} 3$ is not true while $\mathrm{C} 1$ or $\mathrm{C} 2$ is true for the last time period t. What associate with context expressions is Strategy Information; it is explicitly related to application-level behavior. The strategy info describe the actions should be taken when the value of Context Expression becomes true; and the actions can be concluded into dynamic reconfigurations to middleware platform. For instance, usually we use different components in charge of different wireless access solutions, such as 3G/B3G, IEEE 802.1x; when context exchanges from one to another, the components should be dynamically replaced immediately.

\section{Conclusions and Future Work}

This paper presents PURPLE, an adaptive, context-aware and component-based middleware for ubiquitous computing. The use of EFL component platform offers the technique in multi-level reflection, which provides the advantages of flexibility in implementing and extension. Specifically, we discussed the context-awareness mechanism for system-level reconfiguration using strategy control and the working 
process in adaptation and system consistency control. Based on our testing result, we conclude that the overhead by introducing reflection is small enough to be negligible. Finally, the work does not address a number of key issues within ubiquitous computing domain, such as the support for mobility, agent-based programming paradigm. Meanwhile, we would strengthen the collaboration with the development of ubiquitous computing applications to better understand the need and requirement of middleware support.

\section{References}

1. G S Blair, G Coulson, P Robin, M Papathomas. "An Architecture for Next Generation Middleware", In: Proceedings of IFIP International Conference on Distributed Systems Platforms and Open Distributed Processing (Middleware'98). Springer-Verlag, (1998). 191-206

2. Coulson, G., Blair, G.S., Gomes, A.T. et al, "A Reflective Middleware-based Approach to Programmable Networking", Proceedings of 2nd Intl. Workshop on Reflective and Adaptive Middleware (located with ACM/IFIP/USENIX Middleware 2003), Rio de Janeiro, Brazil, June, (2003)

3. Manuel Roman, M Dennis Mickunas, Fabio Kon, R H Campbell. "LegORB and Ubiquitous CORBA", IFIP/ACM Middleware'2000 Workshop on Reflective Middleware. IBM Palisades Executive Conference Center, NY, (2000)

4. Fabio Kon, Manuel Román, Ping Liu et al. "Monitoring, Security, and Dynamic Configuration with the dynamicTAO Reflective ORB", The IFIP/ACM International Conference on Distributed Systems Platforms and Open Distributed Processing. New York, New York, USA, 2000

5. Paul Grace, Gordon S Blair, Sam Samuel. "ReMMoC: A Reflective Middleware to Support Mobile Client Interoperability", Proceedings of International Symposium on Distributed Objects and Applications, volume 2888 of LNCS. Berlin \& Heidelberg, Germany: SpringerVerlag Berlin Heidelberg, (2003). 1170-1187 